\title{
Unfavorable Non-Hodgkin Lymphoma
}

National Cancer Institute

\section{Source}

National Cancer Institute. Unfavorable Non-Hodgkin Lymphoma. NCI Thesaurus. Code C68838.

A non-Hodgkin lymphoma with unfavorable prognosis. 